\title{
An Exploratory Study on the Use of Twitter and Facebook in Tandem
}

\author{
Tasos Spiliotopoulos \\ Madeira-ITI, University of Madeira \\ Funchal, Portugal \\ tspiliot@gmail.com
}

\author{
lan Oakley \\ School of Design and Human Engineering, UNIST \\ Ulsan, South Korea \\ ian.r.oakley@gmail.com
}

\begin{abstract}
The diversity of available Social Network Sites (SNSs) enables people to use multiple services to fulfill their communication needs. Accordingly, this paper argues there is value in studying SNSs simultaneously - that key insights regarding SNS use will be revealed when multiple services are examined together. To demonstrate this point, we present a study of 198 Facebook users with the goal of predicting the likelihood of each being a Twitter user based on their Facebook usage. Exploratory factor analysis on twelve activity metrics collected via the Facebook API led to the identification of five discrete usage dimensions. Of these five dimensions, only those that corresponded to functionality not available in Twitter significantly (and positively) predicted ownership of an account. This result suggests complementary use of the two SNSs based on feature differentiation.
\end{abstract}

Categories and Subject Descriptors

- Human-centered computing Collaborative and social computing theory, concepts and paradigms - Humancentered computing Social networking sites • Humancentered computing User models • Human-centered computing User studies Mathematics of computing Regression analysis - Mathematics of computing Dimensionality reduction - Software and its engineering $\sim$ API languages.

\section{Keywords}

Facebook, Twitter, media selection, technology adoption, social network sites, Facebook API.

\section{INTRODUCTION}

Social Network Sites (SNSs) are fundamentally changing the way humans connect, communicate, and relate to one another. The diversity of available services means that SNSs are often not used in isolation and that users tend to adopt multiple services to fulfill their communication needs. However, despite a diversity of offerings and use, most research on SNSs considers sites in isolation - there are many articles addressing Facebook or Twitter, but little literature that examines the same users' activity on the services together. This paper begins to address this gap in the literature by examining use of Facebook and Twitter in the same participants and with data collected via a survey and the

\footnotetext{
Permission to make digital or hard copies of part or all of this work for personal or classroom use is granted without fee provided that copies are not made or distributed for profit or commercial advantage and that copies bear this notice and the full citation on the first page. Copyrights for third-party components of this work must be honored. For all other uses, contact the Owner/Author.

Copyright is held by the owner/author(s).

British HCI 2015, July 13-17, 2015, Lincoln, United Kingdom

ACM 978-1-4503-3643-7/15/07.
}

http://dx.doi.org/10.1145/2783446.2783620
Facebook Application Programming Interface (API).

Specifically, we collected activity data from the Facebook API because research has found significant discrepancies between selfreported and actual Facebook use [1], and because this data-driven approach can provide broader and more granular data compared to self-report. Thus, we report on 12 variables describing Facebook use and an exploratory factor analysis that identifies dimensions of usage. In order to explore how Twitter users are different from Twitter non-users, we then conduct a binary logistic regression exploring which factors predict ownership of a Twitter account. Showing which aspects of Facebook use predict Twitter use will help shed light on the interplay between the two SNSs

\section{METHOD AND RESULTS}

Participants were recruited with a request to complete an online survey. Approximately $1 / 3$ of participants were recruited through posts on social network sites, $1 / 3$ through posts to online forums, mailing lists and online study repositories, and $1 / 3$ through a Facebook ad campaign. They were directed to a comprehensive study description page that clearly framed the experiment as an academic study and explained the data collection process in detail. The participants then had to explicitly click a link to login with their Facebook credentials and access the survey. The data collected is an extended version of a previously described data set [4], and for the purposes of the current study the survey consisted of demographic information and questions about Facebook usage.

There were a total of 198 usable responses. The participants were 114 males $(57.6 \%)$ and 84 females (42.4\%), with a mean age of 24.6 years old $(\mathrm{SD}=8.78$, range $=14-62$ years old $)$. They came from 30 different countries, with $71(35.9 \%)$ from the USA and $68(34.3 \%)$ from India. The majority of the sample (72.2\%) were full-time students, $24.8 \%$ were employed, and 3\% unemployed. On the days that they use Facebook, participants reported spending a mean of 80.5 (median $=47.5, \mathrm{SD}=90.9)$ minutes on the site, with $42.9 \%$ of the sample $(n=85)$ using Facebook many times per day and $41.4 \%(\mathrm{n}=82)$ every day. Out of the 198 participants in the study, $82(41.4 \%)$ reported using Twitter. On the days that they use Twitter, participants reported spending a mean of 28.7 (median $=15, \mathrm{SD}=45.9$ ) minutes on the service.

An exploratory factor analysis with orthogonal rotation (varimax) was conducted on the 12 user activity variables gathered from the Facebook API in order to identify the dimensions of Facebook usage. Five factors were found with eigenvalues over Kaiser's criterion of 1 and in combination explained $76.05 \%$ of the variance. Examination of the scree plot and unique loadings supported retaining all five factors. We used a cut-off value of 0.6 for factor loadings and no items were discarded. Table 1 depicts the factor loadings after rotation.

A binary logistic regression predicting the likelihood of a Facebook member being also a Twitter member was conducted 
Table 2. Summary of factors describing Facebook usage dimensions

\begin{tabular}{ll}
\hline Item & Factor Loading \\
\hline Text communication $(\boldsymbol{\alpha}=\mathbf{~ 8 2 1 )}$ & .901 \\
Comments made & .885 \\
Likes (to posts, comments etc.) given & .719 \\
Posts made & \\
Photographs $(\boldsymbol{\alpha}=. \mathbf{7 2 3})$ & .843 \\
Photographs posted & .764 \\
Photograph albums created & .649 \\
Photographs tagged in & \\
Profile $(\boldsymbol{\alpha}=. \mathbf{8 3 5})$ & .919 \\
Activities mentioned & .878 \\
Likes (to pages) given & .836 \\
Offline $(\boldsymbol{\alpha}=. \mathbf{6 5 2})$ & .809 \\
Check-ins made & \\
Events attended & .889 \\
Network ( $\alpha=.681)$ & .795 \\
Facebook groups joined & \\
Facebook friends & \\
KMO = .702. $\alpha$ is based on standardized items. & \\
\hline
\end{tabular}

with age, gender, occupation and nationality as controls and the five dimensions of Facebook usage (i.e., the factor scores) as predictors (see Table 2).

\section{DISCUSSION}

Our analysis clearly identifies five discrete dimensions of Facebook usage. The textual communication dimension is associated with the number of posts, comments and likes a user makes, the photographs dimension with the number of photographs, photo albums and tagged photographs associated with the user, the profile dimension with a user's activities (such as playing the guitar) and the number of "likes" to Facebook pages and brands they have made (both visible in a user's profile and typically used to signify their interests), the network dimension with the number of friends and Facebook groups a user engages with, and the offline dimension with the number of location check-ins and offline events associated with the user.

The factor analysis shows that participants' Facebook usage is multidimensional; it breaks down into distinct factors. This suggests people can be classified along these dimensions and into groups with very different usage styles. For instance, members of one group may be very active in sharing photographs and albums, whereas members of another group may use Facebook primarily to organize and support their offline life. We argue that this multidimensional conceptualization of usage behavior provides a more accurate description of individual behaviors than a "compound" measure that just sums up Facebook usage, and can, in turn, be used as meaningful input for further statistical analysis.

Of these five dimensions, the latter two were strong and significant positive predictors of having a Twitter account in addition to being a Facebook user. Interestingly, these two dimensions correspond to features that are not available to Twitter; location check-ins, offline events and Facebook groups. On the other hand, text communication and photograph posting are core features of Twitter and following brands is a common activity. The demographic variables were not found to be significant predictors of having a Twitter account.
Table 1. Binary logistic regression predicting the likelihood of a Facebook user being also a Twitter user

\begin{tabular}{ll}
\hline Measure & $\boldsymbol{\beta}$ \\
\hline Age & -.034 \\
Gender (male) & .389 \\
Occupation (student) & -.600 \\
Nationality (USA) & -.295 \\
\hline Text communication & .052 \\
Photographs & .051 \\
Profile & -.168 \\
Offline & $.622^{* *}$ \\
Network & $.682^{* *}$ \\
\hline Intercept & .812 \\
\hline Nagelkerke $R^{2}=.184 .{ }^{*} p<.05,{ }^{* *} p<.01,{ }^{* * *} p<.001$. All \\
beta coefficients are standardized.
\end{tabular}

These findings suggest that the complementary functionality of the two SNSs can help explain the interplay between them - the presence of differentiated features is a driver for adopting one SNS over another. In contrast, if similar functionality is available in multiple services, users tend to use those features in only a single SNS. In terms of the general claim made in this paper, these results demonstrate that there is observable interplay between the use of the two SNSs and that different usage styles account for nuances in this interplay.

In conclusion, in the volatile and continuously changing landscape of SNSs, this paper argues that studying collections of services together is important for understanding the underlying dynamics that drive social media selection, adoption, use and popularity. Studying usage of multiple SNSs will enable examination of the interplay among SNSs, and be a step towards a more nuanced and complete comprehension of social media in general. Much further work is necessary in this regard; research has highlighted differences between not only users and non-users, but also across different levels of adoption [2] and has identified motivations for use as the main drivers of media selection [3].

\section{REFERENCES}

[1] Junco, R. 2013. Comparing actual and self-reported measures of Facebook use. Computers in Human Behavior 29, 3 (2013), 626-631. DOI = http://dx.doi.org/10.1016/j.chb.2012.11.007.

[2] Lampe, C., Vitak, J., and Ellison, N. 2013. Users and Nonusers: Interactions between Levels of Facebook Adoption and Social Capital. In Proceedings of the 2013 Conference on Computer Supported Cooperative Work. CSCW '13. ACM Press, 809-820. DOI = http://dx.doi.org/10.1145/2441776.2441867.

[3] Papacharissi, Z. and Mendelson, A. 2011. Toward a New(er) Sociability: Uses, Gratifications and Social Capital on Facebook. In S. Papathanassopoulos, ed., Media Perspectives for the 21st Century. Routledge.

[4] Spiliotopoulos, T. and Oakley, I. 2013. Understanding Motivations for Facebook Use: Usage Metrics, Network Structure, and Privacy. In Proceedings of the 2013 ACM Annual Conference on Human Factors in Computing Systems. CHI '13. ACM, $3287-3296$. DOI = http://dx.doi.org/10.1145/2470654.2466449. 